\title{
A sharp Strichartz estimate for the wave equation with data in the energy space
}

Received February 17, 2011 and in revised form August 14, 2011

Abstract. We prove a sharp bilinear estimate for the wave equation from which we obtain the sharp constant in the Strichartz estimate which controls the $L_{t, x}^{4}\left(\mathbb{R}^{5+1}\right)$ norm of the solution in terms of the energy. We also characterise the maximisers.

Keywords. Strichartz estimates, wave equation, sharp constants

\section{Introduction}

For $d \geq 2$, we consider the wave equation $\partial_{t t} u=\Delta u$ on $\mathbb{R}^{d+1}$. Strichartz [29] proved that

$$
\|u\|_{L_{t, x}^{p}\left(\mathbb{R}^{d+1}\right)} \leq C\left(\|u(0)\|_{\dot{H}^{1 / 2}\left(\mathbb{R}^{d}\right)}^{2}+\left\|\partial_{t} u(0)\right\|_{\dot{H}^{-1 / 2}\left(\mathbb{R}^{d}\right)}^{2}\right)^{1 / 2}, \quad p=\frac{2(d+1)}{d-1} .
$$

Here, $\dot{H}^{s}\left(\mathbb{R}^{d}\right)$ denotes the homogeneous Sobolev space with norm

$$
\|f\|_{\dot{H}^{s}\left(\mathbb{R}^{d}\right)}=\left\|(-\Delta)^{s / 2} f\right\|_{L^{2}\left(\mathbb{R}^{d}\right)},
$$

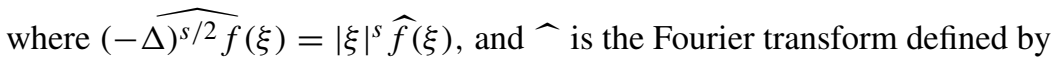

$$
\widehat{f}(\xi)=\int_{\mathbb{R}^{d}} f(x) \exp (-i x \cdot \xi) d x .
$$

Foschi [13] found the sharp constant in (1.1) for $d=3$ and a characterisation of the data $\left(u(0), \partial_{t} u(0)\right)$ for which the constant is attained. That such data exist in higher dimensions is due to Ramos [25].

For $d \geq 3$, by interpolation and Sobolev embedding, (1.1) yields

$$
\|u\|_{L_{t, x}^{p}\left(\mathbb{R}^{d+1}\right)} \leq C\left(\|\nabla u(0)\|_{L^{2}\left(\mathbb{R}^{d}\right)}^{2}+\left\|\partial_{t} u(0)\right\|_{L^{2}\left(\mathbb{R}^{d}\right)}^{2}\right)^{1 / 2}, \quad p=\frac{2(d+1)}{d-2} .
$$

This estimate has found a great deal of application in the nonlinear theory. Indeed, the standard blow-up criterion for the focussing energy-critical equation is written in terms

N. Bez: School of Mathematics, The Watson Building, University of Birmingham, Edgbaston, Birmingham, B15 2TT, England; e-mail: n.bez@bham.ac.uk

K. Rogers: Instituto de Ciencias Matemáticas CSIC-UAM-UC3M-UCM, 28049 Madrid, Spain; e-mail: keith.rogers@icmat.es

Mathematics Subject Classification (2010): Primary 35B45; Secondary 35L05 
of the $L_{t, x}^{p}\left(\mathbb{R}^{d+1}\right)$ norm with $p=2(d+1) /(d-2)$ (see for example [17]). Thus, it seems of interest to know the data which maximise (1.2). That such data exist is due to Bulut [6] (see also [1]).

In this article we prove a sharp bilinear inequality for the one-sided wave propagator $e^{i t \sqrt{-\Delta}}$ given by

$$
e^{i t \sqrt{-\Delta}} f(x)=\frac{1}{(2 \pi)^{d}} \int_{\mathbb{R}^{d}} \widehat{f}(\xi) \exp (i(x \cdot \xi+t|\xi|)) d \xi
$$

The solution to the wave equation can be written as $u=u_{+}+u_{-}$, where

$$
u_{+}(t)=e^{i t \sqrt{-\Delta}} f_{+} \quad \text { and } \quad u_{-}(t)=e^{-i t \sqrt{-\Delta}} f_{-},
$$

and $^{1}$

$$
u(0)=f_{+}+f_{-} \quad \text { and } \quad \partial_{t} u(0)=i \sqrt{-\Delta}\left(f_{+}-f_{-}\right) .
$$

From this bilinear inequality, we will deduce the sharp constant in the energy-Strichartz estimate (1.2) for $d=5$, and characterise the maximising data.

Theorem 1.1. Let $d \geq 2$. Then the inequality

$$
\begin{aligned}
& \left\|e^{i t \sqrt{-\Delta}} f_{1} e^{i t \sqrt{-\Delta}} f_{2}\right\|_{L_{t, x}^{2}\left(\mathbb{R}^{d+1}\right)}^{2} \\
& \quad \leq \mathrm{W}(d, 2) \int_{\mathbb{R}^{2 d}}\left|\widehat{f}_{1}\left(\xi_{1}\right)\right|^{2}\left|\widehat{f}_{2}\left(\xi_{2}\right)\right|^{2}\left|\xi_{1}\right|^{(d-1) / 2}\left|\xi_{2}\right|^{(d-1) / 2}\left(1-\frac{\xi_{1} \cdot \xi_{2}}{\left|\xi_{1}\right|\left|\xi_{2}\right|}\right)^{(d-3) / 2} d \xi_{1} d \xi_{2}
\end{aligned}
$$

holds with constant given by

$$
\mathrm{W}(d, 2)=2^{-(d-1) / 2}(2 \pi)^{-3 d+1}\left|\mathbb{S}^{d-1}\right| .
$$

For $d \geq 3$, the constant is sharp and is attained if and only if

$$
|\xi| \widehat{f}_{j}(\xi)=\exp \left(a|\xi|+b \cdot \xi+c_{j}\right),
$$

where $a, c_{1}, c_{2} \in \mathbb{C}, b \in \mathbb{C}^{d}, \operatorname{Re}(a)<0$ and $|\operatorname{Re}(b)|<-\operatorname{Re}(a)$.

In particular, the constant is attained when $\sqrt{-\Delta} f_{j}(x)=\left(1+|x|^{2}\right)^{-(d+1) / 2}$ for $j=1,2$. In Sections 3 and 4 we prove a $k$-linear generalisation of Theorem 1.1 with sharp constant $\mathrm{W}(d, k)$ for $(d, k) \neq(2,2)$.

Estimates which are similar in spirit, but with different 'null' weights, were proven by Klainerman and Machedon [18, 19, 20], among others. They also conjectured that estimates, for functions with separated angular Fourier supports and with the $L^{2}$ norm on the left-hand side replaced by an $L^{p}$ norm, should hold. For the optimal range of $p$ (modulo the endpoint) this problem was resolved in the remarkable article of Wolff [31] (see Tao [30] for the endpoint), following the pioneering work of Bourgain [5]. The $L^{2}$ version of the null-form conjecture was resolved in [14] and the $L^{p}$-version (modulo the endpoint) in [30, 23, 22].

\footnotetext{
1 In [13] the functions $f_{ \pm}$are defined slightly differently.
} 
When $d=2$, the power of the angular weight is negative, and this estimate was implicit in the work of Barceló [2]. One can calculate that the integral on the right-hand side of (1.3) is unbounded for integrable $f_{1}=\lambda f_{2} \neq 0$.

The power of the angular weight is zero when $d=3$, and in this case the sharp inequality and characterisation of maximisers is due to Foschi [13]. The sharp constant in the Strichartz estimate (1.1) and the characterisation of maximisers follow from this (see [13]).

In contrast with the two-dimensional case, when $d \geq 4$ the estimate (1.3) improves if the interacting waves have overlapping angular Fourier support. In particular, when $d=5$, we will see that Theorem 1.1 is stronger than the sharp energy-Strichartz estimate, which we obtain as a consequence.

Corollary 1.2. Suppose that $\partial_{t t} u=\Delta u$ on $\mathbb{R}^{5+1}$. Then ${ }^{2}$

$$
\|u\|_{L^{4}\left(\mathbb{R}^{5+1}\right)} \leq \frac{1}{(8 \pi)^{1 / 2}}\left(\|\nabla u(0)\|_{L^{2}\left(\mathbb{R}^{5}\right)}^{2}+\left\|\partial_{t} u(0)\right\|_{L^{2}\left(\mathbb{R}^{5}\right)}^{2}\right)^{1 / 2} .
$$

The constant is sharp and is attained if and only if

$$
\left(u(0), \partial_{t} u(0)\right)=\left(0,\left(1+|\cdot|^{2}\right)^{-(d+1) / 2}\right),
$$

with $d=5$, modulo the action of the group generated by

(W1) $u(t, x) \mapsto u\left(t+t_{0}, x+x_{0}\right)$ with $t_{0} \in \mathbb{R}, x_{0} \in \mathbb{R}^{d}$,

(W2) $u(t, x) \mapsto \lambda_{1} u\left(\lambda_{2} t, \lambda_{2} x\right)$ with $\lambda_{1}, \lambda_{2}>0$,

(W3) $u(t, x) \mapsto e^{i \theta_{+}} u_{+}(t, x)+e^{i \theta_{-}} u_{-}(t, x)$ with $\theta_{+}, \theta_{-} \in \mathbb{R}$.

As this is a corollary of Theorem 1.1, the proof relies heavily on the Fourier transform, however the Fourier transform makes no appearance in the final inequality. Indeed the wave equation is often considered as a real equation, and it would be interesting to know if (1.2) could be proven without the use of the complex numbers.

By the conservation of energy, if the initial data is a maximising pair, then $\left(u(t), \partial_{t} u(t)\right)$ must also be a maximising pair. Thus the evolution of the initial data (1.4) can be described in terms (W1)-(W3).

Other well-known symmetries for the wave equation are spatial rotations and Lorentzian boosts:

(W4) $u(t, x) \mapsto u(t, R x)$ with $R \in \mathrm{SO}(d)$,

(W5) $u(t, x) \mapsto u\left(\cosh (a) t+\sinh (a) x_{1}, \cosh (a) x_{1}+\sinh (a) t, x^{\prime}\right)$ with $a \in \mathbb{R}$.

In [13, Theorem 1.7], it is shown that the maximisers for (1.1) with $d=3$ can be obtained from the action of the group generated by (W1)-(W5) on the pair (1.4). Thus, the class of maximisers is larger than that of Corollary 1.2. This is explained by the fact that

$$
\left(\|u(0)\|_{\dot{H}^{1 / 2}\left(\mathbb{R}^{d}\right)}^{2}+\left\|\partial_{t} u(0)\right\|_{\dot{H}^{-1 / 2}\left(\mathbb{R}^{d}\right)}^{2}\right)^{1 / 2}
$$

is invariant under (W5) whereas the energy is not.

We now dedicate some words to the recent history of the problem and the structure of the article. In order to do so, we will need to discuss the closely related Schrödinger

\footnotetext{
2 As usual, the initial data must belong to the energy space which is defined to be the completion of the Schwartz class with respect to the energy norm.
} 
evolution operator $e^{i t \Delta}$ given by

$$
e^{i t \Delta} f(x)=\frac{1}{(2 \pi)^{d}} \int_{\mathbb{R}^{d}} \widehat{f}(\xi) \exp \left(i\left(x \cdot \xi-t|\xi|^{2}\right)\right) d \xi .
$$

Analogously to (1.1), Strichartz [29] proved that

$$
\left\|e^{i t \Delta} f\right\|_{L_{t, x}^{p}\left(\mathbb{R}^{d+1}\right)} \leq C\|f\|_{L^{2}\left(\mathbb{R}^{d}\right)}, \quad p=\frac{2(d+2)}{d},
$$

which followed work of Stein and Tomas on the Fourier extension problem on the unit sphere $\mathbb{S}^{d-1}$.

Some decades later, Kunze [21] proved the existence of maximisers for (1.5) with $d=1$, and Foschi [13] found the maximisers when $d=1,2$. This was reproved via different techniques by Hundertmark-Zharnitsky [16] (see also Bennett et al. [4] for an alternative derivation of the sharp constant when $d=1,2$, using heat-flow methods). Carneiro [7] then developed the ideas of Hundertmark-Zharnitsky in order to prove results analogous to our forthcoming Theorem 2.1 for the Schrödinger operator. That maximisers for (1.5) exist in higher dimensions is due to Shao [26] (see also [24], [10], [3]).

More recently, Duyckaerts, Merle and Roudenko [11] proved that the $L_{t, x}^{p}\left(\mathbb{R}^{d+1}\right)$ norm, with $p=2(d+2) / d$, of the solution to the $L^{2}$-critical nonlinear Schrödinger equation is maximised over data with fixed (small) $L^{2}\left(\mathbb{R}^{d}\right)$ norm. For $d=1,2$, they used the result of Foschi to calculate the size of the maximum norm with some precision. In particular, they showed that the Strichartz norm for the focussing equation with small data is larger than in the linear case. They remark that parts of their proof should be flexible enough to treat the energy-critical Schrödinger and wave equations, and Corollary 1.2 is a step in that direction.

Finally, Christ and Shao [8, 9] proved the existence of maximisers for the original Stein-Tomas extension inequality on the two-dimensional sphere, and that the maximisers $f$ are necessarily smooth and satisfy $|f(x)|=|f(-x)|$. For general compact surfaces and dimensions, Fanelli, Vega and Visciglia [12] obtained the existence of maximisers for the associated extension inequalities up to the endpoint (at which it is also shown that existence is not guaranteed in general). In particular, the result in [12] holds for $p>2(d+2) / d$ when extending on $\mathbb{S}^{d-1}$.

In Section 2, we state our results for the wave propagator in multilinear form and prove Corollary 1.2 and some further corollaries for $d=2,3$. In Section 3, we prove the sharp multilinear inequality, and we characterise the maximisers for this inequality in Section 4. Finally, in Section 5, we revisit the result of Carneiro [7] for the Schrödinger evolution operator.

\section{Main results}

We state our result in full generality (in terms of the multilinearity). In order to write down an expression for the sharp constant $\mathrm{W}(d, k)$ we need the beta function B given by

$$
\mathrm{B}(x, y)=\int_{0}^{1} s^{x-1}(1-s)^{y-1} d s \quad \text { for } x, y>0 .
$$


Theorem 2.1. Suppose that $d, k \geq 2$ and let $\alpha(k)=(d-1)(k-1) / 2-1$. Let $K$ : $\left(\mathbb{R}^{d}\right)^{k} \rightarrow[0, \infty)$ be given by

$$
K(\eta)=\left(\sum_{1 \leq i<j \leq k}\left(\left|\eta_{i}\right|\left|\eta_{j}\right|-\eta_{i} \cdot \eta_{j}\right)\right)^{1 / 2}
$$

for $\eta=\left(\eta_{1}, \ldots, \eta_{k}\right) \in\left(\mathbb{R}^{d}\right)^{k}$. Then the inequality

$$
\left\|\prod_{j=1}^{k} e^{i t \sqrt{-\Delta}} f_{j}\right\|_{L_{t, x}^{2}\left(\mathbb{R}^{d+1}\right)}^{2} \leq \mathrm{W}(d, k) \int_{\mathbb{R}^{k d}} \prod_{j=1}^{k}\left|\widehat{f_{j}}\left(\eta_{j}\right)\right|^{2}\left|\eta_{j}\right| K(\eta)^{2 \alpha(k)} d \eta
$$

holds with constant given by

$$
\mathrm{W}(d, k)=2^{-(d-1) / 2}(2 \pi)^{-3 d+1}\left|\mathbb{S}^{d-1}\right|
$$

if $k=2$, and

$$
\mathrm{W}(d, k)=2^{-(d-1)(k-1) / 2}(2 \pi)^{-d(2 k-1)+1}\left|\mathbb{S}^{d-1}\right|^{k-1} \prod_{j=2}^{k-1} \mathrm{~B}(d-1, \alpha(j)+1)
$$

if $k \geq 3$. Whenever $(d, k) \neq(2,2)$ the constant $\mathrm{W}(d, k)$ is sharp and is attained if and only if

$$
|\xi| \widehat{f}_{j}(\xi)=\exp \left(a|\xi|+b \cdot \xi+c_{j}\right),
$$

where $a, c_{1}, \ldots, c_{k} \in \mathbb{C}, b \in \mathbb{C}^{d}, \operatorname{Re}(a)<0$ and $|\operatorname{Re}(b)|<-\operatorname{Re}(a)$.

Theorem 2.1 was proven by Foschi in the cases where $\alpha(k)=0$. This occurs if and only if $(d, k)$ is either $(2,3)$ or $(3,2)$ and yields the sharp Strichartz estimates for the one-sided operator when $d=2,3$ and a characterisation of the maximisers.

The cases where $\alpha(k)=1$ are also special and this occurs if and only if $(d, k)$ is $(2,5),(3,3)$ or $(5,2)$. We employ a basic yet very useful observation of Carneiro [7] to deduce the following estimates.

Corollary 2.2. In two spatial dimensions,

$$
\left\|e^{i t \sqrt{-\Delta}} f\right\|_{L_{t, x}^{10}\left(\mathbb{R}^{2+1}\right)} \leq\left(\frac{5}{12 \pi^{3}}\right)^{1 / 10}\|f\|_{\dot{H}^{1 / 2}\left(\mathbb{R}^{2}\right)}^{3 / 5}\|f\|_{\dot{H}^{1}\left(\mathbb{R}^{2}\right)}^{2 / 5} .
$$

In three spatial dimensions,

$$
\left\|e^{i t \sqrt{-\Delta}} f\right\|_{L_{t, x}^{6}\left(\mathbb{R}^{3+1}\right)} \leq\left(\frac{3}{16 \pi^{3}}\right)^{1 / 6}\|f\|_{\dot{H}^{1 / 2}\left(\mathbb{R}^{3}\right)}^{1 / 3}\|f\|_{\dot{H}^{1}\left(\mathbb{R}^{3}\right)}^{2 / 3} .
$$

In five spatial dimensions,

$$
\left\|e^{i t \sqrt{-\Delta}} f\right\|_{L_{t, x}^{4}\left(\mathbb{R}^{5+1}\right)} \leq\left(\frac{1}{24 \pi^{2}}\right)^{1 / 4}\|f\|_{\dot{H}^{1}\left(\mathbb{R}^{5}\right)} .
$$

The constants are sharp and are attained if and only if

$$
|\xi| \widehat{f}(\xi)=\exp (a|\xi|+i b \cdot \xi+c),
$$

where $a, c \in \mathbb{C}, b \in \mathbb{R}^{d}$ and $\operatorname{Re}(a)<0$. 
Proof of Corollary 2.2. We have $\alpha(k)=1$. For $f_{1}=\cdots=f_{k}=f$, the integral on the right-hand side of (2.1) can be written as

$$
\int_{\mathbb{R}^{k d}} \prod_{\ell=1}^{k}\left|\widehat{f}\left(\eta_{\ell}\right)\right|^{2}\left|\eta_{\ell}\right| K(\eta)^{2 \alpha(k)} d \eta=\mathrm{I}-\mathrm{II},
$$

where

$$
\begin{aligned}
\mathrm{I} & =\sum_{1 \leq i<j \leq k} \int_{\mathbb{R}^{k d}} \prod_{\ell=1}^{k}\left|\widehat{f}\left(\eta_{\ell}\right)\right|^{2}\left|\eta_{\ell}\right|\left|\eta_{i}\right|\left|\eta_{j}\right| d \eta=\frac{k(k-1)}{2}(2 \pi)^{k d}\|f\|_{\dot{H}^{1 / 2}\left(\mathbb{R}^{d}\right)}^{2(k-2)}\|f\|_{\dot{H}^{1}\left(\mathbb{R}^{d}\right)}^{4}, \\
\mathrm{II} & =\sum_{1 \leq i<j \leq k} \int_{\mathbb{R}^{k d}} \prod_{\ell=1}^{k}\left|\widehat{f}\left(\eta_{\ell}\right)\right|^{2}\left|\eta_{\ell}\right| \eta_{i} \cdot \eta_{j} d \eta .
\end{aligned}
$$

As in [7], by writing $\xi=\left(\xi_{1}, \ldots, \xi_{d}\right)$, we have

$$
\int_{\mathbb{R}^{2 d}}\left|\widehat{f}\left(\eta_{i}\right)\right|^{2}\left|\eta_{i}\right|\left|\widehat{f}\left(\eta_{j}\right)\right|^{2}\left|\eta_{j}\right| \eta_{i} \cdot \eta_{j} d \eta_{i} d \eta_{j}=\sum_{m=1}^{d}\left(\int_{\mathbb{R}^{d}}|\widehat{f}(\xi)|^{2}|\xi| \xi_{m} d \xi\right)^{2} \geq 0
$$

so that by Theorem 2.1,

$$
\begin{aligned}
\left\|e^{i t \sqrt{-\Delta}} f\right\|_{L_{t, x}^{2 k}\left(\mathbb{R}^{d+1}\right)}^{2 k} & \leq \mathrm{W}(d, k)(\mathrm{I}-\mathrm{II}) \\
& \leq\left[\frac{k(k-1)}{2}(2 \pi)^{k d} \mathrm{~W}(d, k)\right]\|f\|_{\dot{H}^{1 / 2}\left(\mathbb{R}^{d}\right)}^{2(k-2)}\|f\|_{\dot{H}^{1}\left(\mathbb{R}^{d}\right)}^{4}
\end{aligned}
$$

with equality at each inequality for the functions with radial modulus given by

$$
\widehat{f}(\xi)=|\xi|^{-1} \exp (a|\xi|+b \cdot \xi+c),
$$

where $a, c \in \mathbb{C}, b \in \mathbb{C}^{d}$ and $\operatorname{Re}(b)=0$.

It remains to characterise the maximisers, that is, to prove that when $b \in \mathbb{C}^{d}$ with $\operatorname{Re}(b) \neq 0$, the quantity II is nonzero. By a rotation we can suppose that

$$
\widehat{f}(\xi)=|\xi|^{-1} \exp (a|\xi|+b \cdot \xi+c),
$$

where $a, c \in \mathbb{C}, b \in \mathbb{C}^{d}, \operatorname{Re}(a)<0, \operatorname{Re}(b)=\left(b_{1}, 0, \ldots, 0\right)$ and $b_{1}>0$. By (2.2), it suffices to prove that

$$
\left(\int_{\mathbb{R}^{d}}|\widehat{f}(\xi)|^{2}|\xi| \xi_{1} d \xi\right)^{2}>0
$$

which is the same thing as proving

$$
\left(\int_{\mathbb{R}^{d}}|\xi|^{-1} \exp \left(2 \operatorname{Re}(a)|\xi|+2 b_{1} \xi_{1}+2 \operatorname{Re}(c)\right) \xi_{1} d \xi\right)^{2}>0
$$


Writing $\mathbb{R}_{+}^{d}=\left\{\xi \in \mathbb{R}^{d}: \xi_{1} \geq 0\right\}$, the left-hand side of this inequality is equal to

$$
\left(\int_{\mathbb{R}_{+}^{d}}|\xi|^{-1} \exp (2 \operatorname{Re}(a)|\xi|+2 \operatorname{Re}(c))\left(\exp \left(2 b_{1} \xi_{1}\right)-\exp \left(-2 b_{1} \xi_{1}\right)\right) \xi_{1} d \xi\right)^{2},
$$

which is positive, and so we are done.

We conclude this section by showing how Corollary 1.2 can be deduced from Corollary 2.2 following Foschi [13].

Proof of Corollary 1.2. By Corollary 2.2, we have the sharp inequality

$$
\left\|u_{+}\right\|_{L_{t, x}^{4}\left(\mathbb{R}^{5+1}\right)} \leq\left(24 \pi^{2}\right)^{-1 / 4}\left\|\nabla f_{+}\right\|_{L^{2}\left(\mathbb{R}^{5}\right)}
$$

with equality if $|\xi| \widehat{f_{+}}(\xi)=\exp (-|\xi|)$, and by the same argument we also have

$$
\left\|u_{-}\right\|_{L_{t, x}^{4}\left(\mathbb{R}^{5+1}\right)} \leq\left(24 \pi^{2}\right)^{-1 / 4}\left\|\nabla f_{-}\right\|_{L^{2}\left(\mathbb{R}^{5}\right)}
$$

with equality if $|\xi| \widehat{f_{-}}(\xi)=\exp (-|\xi|)$.

The space-time Fourier transforms of $u_{+}^{2}, u_{-}^{2}$ and $u_{+} u_{-}$have disjoint supports and therefore

$$
\begin{aligned}
\|u\|_{L_{t, x}^{4}\left(\mathbb{R}^{5+1}\right)}^{4} & =\left\|u_{+}^{2}+u_{-}^{2}+2 u_{+} u_{-}\right\|_{L_{t, x}^{2}\left(\mathbb{R}^{5+1}\right)}^{2} \\
& =\left\|u_{+}\right\|_{L_{t, x}^{4}\left(\mathbb{R}^{5+1}\right)}^{4}+\left\|u_{-}\right\|_{L_{t, x}^{4}\left(\mathbb{R}^{5+1}\right)}^{4}+4\left\|u_{+} u_{-}\right\|_{L_{t, x}^{2}\left(\mathbb{R}^{5+1}\right)}^{2} .
\end{aligned}
$$

By the Cauchy-Schwarz inequality,

$$
\left\|u_{+} u_{-}\right\|_{L_{t, x}^{2}\left(\mathbb{R}^{5+1}\right)}^{2} \leq\left\|u_{+}\right\|_{L_{t, x}^{4}\left(\mathbb{R}^{5+1}\right)}^{2}\left\|u_{-}\right\|_{L_{t, x}^{4}\left(\mathbb{R}^{5+1}\right)}^{2}
$$

with equality if and only if $\left|u_{+}\right|=\lambda\left|u_{-}\right|$for some $\lambda>0$. We now apply the elementary inequality

$$
2\left(X^{2}+Y^{2}+4 X Y\right) \leq 3(X+Y)^{2},
$$

which holds for all real numbers $X$ and $Y$, with equality if and only if $X=Y$, to obtain

$$
\|u\|_{L_{t, x}^{4}\left(\mathbb{R}^{5+1}\right)}^{4} \leq \frac{3}{2}\left(\left\|u_{+}\right\|_{L_{t, x}^{4}\left(\mathbb{R}^{5+1}\right)}^{2}+\left\|u_{-}\right\|_{L_{t, x}^{4}\left(\mathbb{R}^{5+1}\right)}^{2}\right)^{2} .
$$

Combining this with (2.3) and (2.4), it follows that

$$
\|u\|_{L_{t, x}^{4}\left(\mathbb{R}^{5+1}\right)}^{4} \leq \frac{1}{16 \pi^{2}}\left(\left\|\nabla f_{+}\right\|_{L^{2}\left(\mathbb{R}^{5}\right)}^{2}+\left\|\nabla f_{-}\right\|_{L^{2}\left(\mathbb{R}^{5}\right)}^{2}\right)^{2} .
$$

By the parallelogram law,

$$
\left\|\nabla f_{+}\right\|_{L^{2}\left(\mathbb{R}^{5}\right)}^{2}+\left\|\nabla f_{-}\right\|_{L^{2}\left(\mathbb{R}^{5}\right)}^{2}=\frac{1}{2}\left(\|\nabla u(0)\|_{L^{2}\left(\mathbb{R}^{5}\right)}^{2}+\left\|\partial_{t} u(0)\right\|_{L^{2}\left(\mathbb{R}^{5}\right)}^{2},\right.
$$

so that

$$
\|u\|_{L_{t, x}^{4}\left(\mathbb{R}^{5+1}\right)}^{4} \leq \frac{1}{64 \pi^{2}}\left(\|\nabla u(0)\|_{L^{2}\left(\mathbb{R}^{5}\right)}^{2}+\left\|\partial_{t} u(0)\right\|_{L^{2}\left(\mathbb{R}^{5}\right)}^{2}\right)^{2},
$$


with equality when $\left(u(0), \partial_{t} u(0)\right)$ is such that $|\xi| \widehat{f_{+}}(\xi)=|\xi| \widehat{f_{-}}(\xi)=\exp (-|\xi|)$ (because then $\left.\left|u_{+}\right|=\left|u_{-}\right|\right)$.

It remains to characterise the maximisers. It follows from Corollary 2.2 and the above argument that we have equality in (2.7) if and only if

$$
|\xi| \widehat{f_{+}}(\xi)=\exp \left(a_{+}|\xi|+i b_{+} \cdot \xi+c_{+}\right), \quad|\xi| \widehat{f_{-}}(\xi)=\exp \left(a_{-}|\xi|+i b_{-} \cdot \xi+c_{-}\right),
$$

and

$$
\left|u_{+}(t, x)\right|=\left|u_{-}(t, x)\right| \quad \text { for almost every }(t, x) \in \mathbb{R} \times \mathbb{R}^{5},
$$

where $a_{+}, a_{-}, c_{+}, c_{-} \in \mathbb{C}, b_{+}, b_{-} \in \mathbb{R}^{5}$ and $\operatorname{Re}\left(a_{+}\right), \operatorname{Re}\left(a_{-}\right)<0$. We will see that this is true if and only if $a_{+}=\overline{a_{-}}, b_{+}=b_{-}$and $\operatorname{Re}\left(c_{+}\right)=\operatorname{Re}\left(c_{-}\right)$.

To this end we define $\Lambda_{a, b, c}$ by

$$
\Lambda_{a, b, c}(t, x)=\frac{1}{(2 \pi)^{5}}\left|\exp (c) \int_{\mathbb{R}^{5}} \exp (i(b+x) \cdot \xi+(a+i t)|\xi|) \frac{d \xi}{|\xi|}\right| .
$$

As $\left|u_{+}\right|=\Lambda_{a_{+}, b_{+}, c_{+}}$and $\left|u_{-}\right|=\left|\overline{u_{-}}\right|=\Lambda_{\overline{a_{-}, b_{-}, \bar{c}_{-}}}$, and these functions are continuous, we see by (2.8) that

$$
\Lambda_{a_{+}, b_{+}, c_{+}}(t, x)=\Lambda_{\overline{a_{-}}, b_{-}, \overline{c_{-}}}(t, x) \quad \text { for each }(t, x) \in \mathbb{R} \times \mathbb{R}^{5} .
$$

As in [13], we claim that knowledge of $\Lambda_{a, b, c}$ uniquely determines $a, b$ and $\operatorname{Re}(c)$. Given (2.9), it would then follow that $a_{+}=\overline{a_{-}}, b_{+}=b_{-}$and $\operatorname{Re}\left(c_{+}\right)=\operatorname{Re}\left(c_{-}\right)$.

Firstly, we note that

$$
\Lambda_{a, b, c}(t, x) \leq \frac{\exp (\operatorname{Re}(c))}{(2 \pi)^{5}} \int_{\mathbb{R}^{5}} \exp (\operatorname{Re}(a)|\xi|) \frac{d \xi}{|\xi|}=\Lambda_{a, b, c}(-\operatorname{Im}(a),-b),
$$

so we see that $\Lambda_{a, b, c}$ attains its maximum at $(-\operatorname{Im}(a),-b)$ and nowhere else. $\operatorname{Thus,} \operatorname{Im}(a)$ and $b$ are uniquely determined. Secondly,

$$
\Lambda_{a, b, c}(t-\operatorname{Im}(a),-b)=\frac{\exp (\operatorname{Re}(c))}{(2 \pi)^{5}}\left|\int_{\mathbb{R}^{5}} \exp ((\operatorname{Re}(a)+i t)|\xi|) \frac{d \xi}{|\xi|}\right|=C_{0} \frac{\exp (\operatorname{Re}(c))}{|\operatorname{Re}(a)+i t|^{4}}
$$

where $C_{0}$ is an absolute constant. Thus,

$$
C_{0} \Lambda_{a, b, c}(t-\operatorname{Im}(a),-b)^{-1}=\exp (-\operatorname{Re}(c))\left(\operatorname{Re}(a)^{2}+t^{2}\right)^{2},
$$

which is a polynomial in $t$. Since the coefficient of $t^{4}$ is $\exp (-\operatorname{Re}(c))$ we have determined $\operatorname{Re}(c)$, and since the constant term is $\exp (-\operatorname{Re}(c)) \operatorname{Re}(a)^{4}$ we have then determined $\operatorname{Re}(a)$.

It remains to prove that these maximisers can be obtained from

$$
\left(u(0, x), \partial_{t} u(0, x)\right)=\left(0,\left(1+|x|^{2}\right)^{-3}\right)
$$

under the action of (W1)-(W3) as defined in the introduction. It is easy to calculate that the ratio

$$
\|u\|_{L_{t, x}^{4}\left(\mathbb{R}^{5+1}\right)}\left(\|\nabla u(0)\|_{L^{2}\left(\mathbb{R}^{5}\right)}^{2}+\left\|\partial_{t} u(0)\right\|_{L^{2}\left(\mathbb{R}^{5}\right)}^{2}\right)^{-1 / 2}
$$


is preserved under the action of (W1) and (W2). Appealing to (2.5) and (2.6) we see that the ratio is also preserved under (W3). We remark that this final invariance does not hold in general for Strichartz inequalities.

Taking Fourier transforms of the data in (2.10) we obtain

$$
\left.\widehat{(u(0)}(\xi), \widehat{\partial_{t} u(0)}(\xi)\right)=\left(0, c_{0} \exp (-|\xi|)\right)
$$

for some $c_{0}>0$ (see for example [27, p. 61]). Consequently,

$$
\left(|\xi| \widehat{f_{+}}(\xi),|\xi| \widehat{f_{-}}(\xi)\right)=\left(\frac{1}{2 i} c_{0} \exp (-|\xi|),-\frac{1}{2 i} c_{0} \exp (-|\xi|)\right) .
$$

In general, the data $\widehat{f_{ \pm}}(\xi)$ transforms to

$$
\exp \left( \pm i t_{0}|\xi|+i x_{0} \cdot \xi\right) \widehat{f_{ \pm}}(\xi), \quad \lambda_{1} \lambda_{2}^{-5} \widehat{f_{ \pm}}\left(\lambda_{2}^{-1} \xi\right), \quad \exp \left(i \theta_{ \pm}\right) \widehat{f_{ \pm}}(\xi)
$$

under the action of (W1), (W2), (W3), respectively. It is now straightforward to check that the pair (2.11) transforms under the action of (W1)-(W3) to

$$
\left(|\xi| \widehat{f_{+}}(\xi),|\xi| \widehat{f_{-}}(\xi)\right)=\left(\exp \left(a|\xi|+i b \cdot \xi+c_{+}\right), \exp \left(\bar{a}|\xi|+i b \cdot \xi+c_{-}\right)\right)
$$

where $\operatorname{Re}\left(c_{+}\right)=\operatorname{Re}\left(c_{-}\right)$, and so we are done.

Remark 2.3. Foschi [13] combined similar arguments with his sharp estimate for the one-sided operator,

$$
\left\|e^{i t \sqrt{-\Delta}} f\right\|_{L_{t, x}^{6}\left(\mathbb{R}^{2+1}\right)} \leq\left(\frac{1}{2 \pi}\right)^{1 / 6}\|f\|_{\dot{H}^{1 / 2}\left(\mathbb{R}^{2}\right)}
$$

to prove that solutions to the wave equation satisfy

$$
\|u\|_{L_{t, x}^{6}\left(\mathbb{R}^{2+1}\right)} \leq\left(\frac{25}{64 \pi}\right)^{1 / 6}\left(\|u(0)\|_{\dot{H}^{1 / 2}\left(\mathbb{R}^{2}\right)}^{2}+\left\|\partial_{t} u(0)\right\|_{\dot{H}^{-1 / 2}\left(\mathbb{R}^{2}\right)}^{2}\right)^{1 / 2}
$$

He also claimed that the constant in (2.12) is attained by the initial data

$$
\left(u(0, x), \partial_{t} u(0, x)\right)=\left(\left(1+|x|^{2}\right)^{-1 / 2}, 0\right),
$$

modulo the action of a group of symmetries, but this appears to be false. In the proof of (2.12), the inequality

$$
\left|\left\langle u_{+}^{3}, u_{+}^{2} u_{-}\right\rangle_{t, x}\right| \leq\left\|u_{+}^{3}\right\|_{L_{t, x}^{2}\left(\mathbb{R}^{2+1}\right)}\left\|u_{+}^{2} u_{-}\right\|_{L_{t, x}^{2}\left(\mathbb{R}^{2+1}\right)}
$$

is used and it is a strict inequality for such data. 


\section{Proof of Theorem 2.1-the sharp inequality}

Define the Fourier transform $\sim$ in space and time by

$$
\tilde{f}(\tau, \xi)=\int_{\mathbb{R}^{d+1}} f(t, x) \exp (-i(t \tau+x \cdot \xi)) d t d x .
$$

Writing

$$
u_{k}(t, x)=\prod_{j=1}^{k} e^{i t \sqrt{-\Delta}} f_{j}(x)
$$

by Plancherel's theorem we have

$$
\left\|\prod_{j=1}^{k} e^{i t \sqrt{-\Delta}} f_{j}\right\|_{L_{t, x}^{2}\left(\mathbb{R}^{d+1}\right)}^{2}=(2 \pi)^{-(d+1)}\left\|\tilde{u}_{k}\right\|_{L_{\tau, \xi}^{2}\left(\mathbb{R}^{d+1}\right)}^{2}
$$

It is easy to see that

$$
\left(e^{i t \sqrt{-\Delta}} f_{j}\right) \sim(\tau, \xi)=2 \pi \delta(\tau-|\xi|) \widehat{f_{j}}(\xi),
$$

so that defining $F$ by $\widehat{F}(\eta)=\prod_{j=1}^{k}\left|\eta_{j}\right|^{1 / 2} \widehat{f_{j}}\left(\eta_{j}\right)$, where $\eta=\left(\eta_{1}, \ldots, \eta_{k}\right)$, we have

$$
\tilde{u}_{k}(\tau, \xi)=\frac{1}{(2 \pi)^{d(k-1)-1}} \int_{\mathbb{R}^{k d}} \frac{\widehat{F}(\eta)}{\prod_{j=1}^{k}\left|\eta_{j}\right|^{1 / 2}} \delta\left(\tau-\sum_{j=1}^{k}\left|\eta_{j}\right|\right) \delta\left(\xi-\sum_{j=1}^{k} \eta_{j}\right) d \eta .
$$

By the Cauchy-Schwarz inequality, this implies that

$$
\left|\tilde{u}_{k}(\tau, \xi)\right|^{2} \leq \frac{I_{k}(\tau, \xi)}{(2 \pi)^{2 d(k-1)-2}} \int_{\mathbb{R}^{k d}}|\widehat{F}(\eta)|^{2} K(\eta)^{2 \alpha(k)} \delta\left(\tau-\sum_{j=1}^{k}\left|\eta_{j}\right|\right) \delta\left(\xi-\sum_{j=1}^{k} \eta_{j}\right) d \eta,
$$

where $\alpha(k)=(d-1)(k-1) / 2-1$ and

$$
I_{k}(\tau, \xi)=\int_{\mathbb{R}^{k d}} \frac{1}{K(\eta)^{2 \alpha(k)} \prod_{j=1}^{k}\left|\eta_{j}\right|} \delta\left(\tau-\sum_{j=1}^{k}\left|\eta_{j}\right|\right) \delta\left(\xi-\sum_{j=1}^{k} \eta_{j}\right) d \eta .
$$

Crucially, on the intersection of the supports of the delta measures we have

$$
2 K(\eta)^{2}=\left(\sum_{j=1}^{k}\left|\eta_{j}\right|\right)^{2}-\left|\sum_{j=1}^{k} \eta_{j}\right|^{2}=\tau^{2}-|\xi|^{2}
$$

and therefore

$$
I_{k}(\tau, \xi)=2^{\alpha(k)}\left(\tau^{2}-|\xi|^{2}\right)^{-\alpha(k)} \widetilde{I}_{k}(\tau, \xi),
$$

where

$$
\tilde{I}_{k}(\tau, \xi)=\int_{\mathbb{R}^{k d}} \frac{1}{\prod_{j=1}^{k}\left|\eta_{j}\right|} \delta\left(\tau-\sum_{j=1}^{k}\left|\eta_{j}\right|\right) \delta\left(\xi-\sum_{j=1}^{k} \eta_{j}\right) d \eta .
$$

The following lemma was proven by Foschi in the cases $(d, k)=(2,3)$ and $(3,2)$. We generalise his argument by induction. 
Lemma 3.1. For each $(\tau, \xi)$ with $|\xi|<\tau$, we have

$$
\tilde{I}_{2}(\tau, \xi)=\left(\tau^{2}-|\xi|^{2}\right)^{\alpha(2)} \frac{\left|\mathbb{S}^{d-1}\right|}{2^{d-2}},
$$

and for $k \geq 3$,

$$
\widetilde{I}_{k}(\tau, \xi)=\left(\tau^{2}-|\xi|^{2}\right)^{\alpha(k)} \frac{\left|\mathbb{S}^{d-1}\right|^{k-1}}{2^{2 \alpha(k)+1}}\left(\prod_{j=2}^{k-1} \mathrm{~B}(d-1, \alpha(j)+1)\right) .
$$

Proof. We begin by recording certain invariances of $\widetilde{I}_{k}$. Note that $\widetilde{I}_{k}$ is the $k$-fold convolution of $\mu$, where

$$
\mu(\tau, \xi)=|\xi|^{-1} \delta(\tau-|\xi|)=2 \delta(\varrho(\tau, \xi)) \chi_{\tau>0}
$$

and $\varrho: \mathbb{R}^{d} \rightarrow \mathbb{R}$ is the Minkowski form given by $\varrho(\tau, \xi)=\tau^{2}-|\xi|^{2}$. This is invariant under Lorentz transformations:

$$
\varrho\left(T_{v}(\tau, \xi)\right)=\varrho(\tau, \xi)
$$

where the Lorentz transformation $T_{v}$ is given by

$$
T_{v}\left[\begin{array}{l}
\tau \\
\xi
\end{array}\right]=\left[\begin{array}{cc}
\gamma & -\gamma v^{t} \\
-\gamma v & I_{d}+\frac{\gamma-1}{|v|^{2}} v v^{t}
\end{array}\right]\left[\begin{array}{l}
\tau \\
\xi
\end{array}\right]=\left[\begin{array}{c}
\gamma(\tau-v \cdot \xi) \\
\xi+\left(\frac{\gamma-1}{|v|^{2}} v \cdot \xi-\gamma \tau\right) v
\end{array}\right]
$$

for $v \in \mathbb{R}^{d}$ such that $|v|<1$, and $\gamma=\left(1-|v|^{2}\right)^{-1 / 2}$. Since $\left|\operatorname{det} T_{v}\right|=1$ it follows that the $k$-fold convolution of $\mu$ is also invariant under each $T_{v}$. Taking $v=-\xi / \tau$, as we may, we have $\gamma=\left(\tau^{2}-|\xi|^{2}\right)^{-1 / 2} \tau$, and

$$
T_{v}\left[\begin{array}{c}
\left(\tau^{2}-|\xi|^{2}\right)^{1 / 2} \\
0
\end{array}\right]=\left[\begin{array}{c}
\tau \\
-\tau v
\end{array}\right]=\left[\begin{array}{l}
\tau \\
\xi
\end{array}\right]
$$

so that

$$
\widetilde{I}_{k}(\tau, \xi)=\widetilde{I}_{k}\left(\left(\tau^{2}-|\xi|^{2}\right)^{1 / 2}, 0\right) \text {. }
$$

Furthermore, by a simple change of variables and homogeneity, for each $\lambda>0$,

$$
\widetilde{I}_{k}(\lambda \tau, \lambda \xi)=\lambda^{2 \alpha(k)} \widetilde{I}_{k}(\tau, \xi)
$$

where $\alpha(k)=(d-1)(k-1) / 2-1$, which combined with (3.3) yields

$$
\widetilde{I}_{k}(\tau, \xi)=\left(\tau^{2}-|\xi|^{2}\right)^{\alpha(k)} \widetilde{I}_{k}(1,0) .
$$

Now we are able to compute the desired expression for $\tilde{I}_{2}(\tau, \xi)$ by a direct computation. By (3.4) we get

$$
\begin{aligned}
\tilde{I}_{2}(\tau, \xi) & =\left(\tau^{2}-|\xi|^{2}\right)^{\alpha(2)} \tilde{I}_{2}(1,0) \\
& =\left(\tau^{2}-|\xi|^{2}\right)^{\alpha(2)} \int_{\mathbb{R}^{2 d}} \delta\left(1-\left|\eta_{1}\right|-\left|\eta_{2}\right|\right) \delta\left(-\eta_{1}-\eta_{2}\right) \frac{d \eta_{1} d \eta_{2}}{\left|\eta_{1}\right|\left|\eta_{2}\right|} \\
& =\left(\tau^{2}-|\xi|^{2}\right)^{\alpha(2)} \int_{\mathbb{R}^{d}} \delta\left(1-2\left|\eta_{1}\right|\right) \frac{d \eta_{1}}{\left|\eta_{1}\right|^{2}},
\end{aligned}
$$


and hence, by integration in polar coordinates,

$$
\widetilde{I}_{2}(\tau, \xi)=\left(\tau^{2}-|\xi|^{2}\right)^{\alpha(2)} \frac{\left|\mathbb{S}^{d-1}\right|}{2^{d-2}}
$$

as required.

Using (3.4), for $k \geq 3$, observe that

$$
\begin{aligned}
\tilde{I}_{k}(\tau, \xi) & =\left(\tau^{2}-|\xi|^{2}\right)^{\alpha(k)} \tilde{I}_{k}(1,0) \\
& =\left(\tau^{2}-|\xi|^{2}\right)^{\alpha(k)} \int_{\mathbb{R}^{d}}\left(\int_{\mathbb{R}^{(k-1) d}} \delta\left(1-\sum_{j=1}^{k}\left|\eta_{j}\right|\right) \delta\left(-\sum_{j=1}^{k} \eta_{j}\right) \frac{d \eta_{2} \cdots d \eta_{k}}{\left|\eta_{2}\right| \cdots\left|\eta_{k}\right|}\right) \frac{d \eta_{1}}{\left|\eta_{1}\right|}
\end{aligned}
$$

and therefore

$$
\widetilde{I}_{k}(\tau, \xi)=\left(\tau^{2}-|\xi|^{2}\right)^{\alpha(k)} \int_{\left|\eta_{1}\right| \leq 1 / 2} \widetilde{I}_{k-1}\left(1-\left|\eta_{1}\right|,-\eta_{1}\right) \frac{d \eta_{1}}{\left|\eta_{1}\right|} .
$$

Using (3.5) and (3.6) it follows that

$$
\begin{aligned}
\widetilde{I}_{3}(\tau, \xi) & =\left(\tau^{2}-|\xi|^{2}\right)^{\alpha(3)} \frac{\left|\mathbb{S}^{d-1}\right|}{2^{d-2}} \int_{|\eta| \leq 1 / 2}\left(1-2\left|\eta_{1}\right|\right)^{\alpha(2)} \frac{d \eta_{1}}{\left|\eta_{1}\right|} \\
& =\left(\tau^{2}-|\xi|^{2}\right)^{\alpha(3)} \frac{\left|\mathbb{S}^{d-1}\right|^{2}}{2^{d-2}} \int_{0}^{1 / 2}(1-2 r)^{\alpha(2)} r^{d-2} d r .
\end{aligned}
$$

From this, (3.6) and induction it follows that

$$
\widetilde{I}_{k}(\tau, \xi)=\left(\tau^{2}-|\xi|^{2}\right)^{\alpha(k)} \frac{\left|\mathbb{S}^{d-1}\right|^{k-1}}{2^{d-2}} \prod_{j=2}^{k-1}\left(\int_{0}^{1 / 2}(1-2 r)^{\alpha(j)} r^{d-2} d r\right),
$$

which gives the desired formula for $\widetilde{I}_{k}(\tau, \xi)$ by a simple change of variables.

Combining Lemma 3.1 with (3.2) we obtain

$$
I_{2}(\tau, \xi)=2^{-(d-1) / 2}\left|\mathbb{S}^{d-1}\right|
$$

and

$$
I_{k}(\tau, \xi)=2^{-(\alpha(k)+1)}\left|\mathbb{S}^{d-1}\right|^{k-1} \prod_{j=2}^{k-1} \mathrm{~B}(d-1, \alpha(j)+1)
$$

if $k \geq 3$. Substituting into (3.1), integrating over $(\tau, \xi)$, and applying Fubini and Plancherel's theorems, we get

$$
\left\|u_{k}\right\|_{L_{t, x}^{2}\left(\mathbb{R}^{d+1}\right)}^{2}=(2 \pi)^{-(d+1)}\left\|\widetilde{u}_{k}\right\|_{L_{\tau, \xi}^{2}\left(\mathbb{R}^{d+1}\right)}^{2} \leq \mathrm{W}(d, k) \int_{\mathbb{R}^{k d}}|\widehat{F}(\eta)|^{2} K(\eta)^{2 \alpha(k)} d \eta,
$$

as required. 
We note that if $\left|\eta_{j}\right| \widehat{f_{j}}\left(\eta_{j}\right)=\exp \left(a\left|\eta_{j}\right|+b \cdot \eta_{j}+c_{j}\right)$, where $a, c_{1}, \ldots, c_{k} \in \mathbb{C}, b \in \mathbb{C}^{d}$ with $\operatorname{Re}(a)<0$ and $|\operatorname{Re}(b)|<-\operatorname{Re}(a)$, it follows that

$$
\widehat{F}(\eta)=\exp \left(a \tau+b \cdot \xi+\sum_{j=1}^{k} c_{j}\right) \frac{1}{\prod_{j=1}^{k}\left|\eta_{j}\right|^{1 / 2}}
$$

on the support of the delta measures. Hence, for such $f_{j}$ there is equality in (3.1) and so the constant $\mathrm{W}(d, k)$ is sharp whenever $(d, k) \neq(2,2)$. In the next section we show that there are no further maximisers, following the approach of Foschi [13].

\section{Proof of Theorem 2.1-characterisation of the maximisers}

There is equality in (3.1) if and only if there exists a scalar function $\Lambda$ such that

$$
K(\eta)^{\alpha(k)} \widehat{F}(\eta)=\Lambda(\tau, \xi) K(\eta)^{-\alpha(k)} \prod_{j=1}^{k}\left|\eta_{j}\right|^{-1 / 2}
$$

almost everywhere on the intersection of the supports of the delta measures. Write $g_{j}\left(\eta_{j}\right)=\left|\eta_{j}\right| \widehat{f_{j}}\left(\eta_{j}\right)$ for all $j=1, \ldots, k$ and $G(\tau, \xi)=\left(\tau^{2}-|\xi|^{2}\right)^{-\alpha(k)} \Lambda(\tau, \xi)$. Then (4.1) implies that

$$
\prod_{j=1}^{k} g_{j}\left(\eta_{j}\right)=G\left(\sum_{j=1}^{k}\left|\eta_{j}\right|, \sum_{j=1}^{k} \eta_{j}\right) \quad \text { for almost every }\left(\eta_{1}, \ldots, \eta_{k}\right) \in\left(\mathbb{R}^{d}\right)^{k} .
$$

Since the right-hand side of (4.2) is symmetric in $\eta_{j}$ and $\eta_{\ell}$ it follows that $g_{j}=\lambda g_{\ell}$ for some $\lambda \in \mathbb{C}$. By normalising, we can thus assume that $g_{1}=\cdots=g_{k}=g$.

Note that when $f_{1}=\cdots=f_{k}=f$ and $(d, k) \neq(2,2)$ the right-hand side of $(2.1)$ is comparable to

$$
\|f\|_{\dot{H}^{1 / 2}\left(\mathbb{R}^{d}\right)}^{2(k-2)} \int_{\mathbb{R}^{d}} \int_{\mathbb{R}^{d}}\left|\widehat{f}\left(\eta_{1}\right)\right|^{2}\left|\widehat{f}\left(\eta_{2}\right)\right|^{2}\left|\eta_{1}\right|^{\alpha(k)+1}\left|\eta_{2}\right|^{\alpha(k)+1}\left(1-\eta_{1}^{\prime} \cdot \eta_{2}^{\prime}\right)^{\alpha(k)} d \eta_{1} d \eta_{2},
$$

where $\eta_{j}^{\prime}=\left|\eta_{j}\right|^{-1} \eta_{j}$. We claim that when this quantity is finite then $g$ and $G$ satisfying (4.2) are continuous, where $g(\eta)=|\eta| \widehat{f}(\eta)$. To see this, first note that the finiteness of (4.3) implies that $g$ is locally integrable. Indeed, if $k=2$ and $B$ is any euclidean ball centred at the origin then, by the Cauchy-Schwarz inequality and the finiteness of (4.3),

$$
\begin{aligned}
\left(\int_{B}|g(\eta)| d \eta\right)^{2} & =\int_{B} \int_{B}\left|g\left(\eta_{1}\right)\right|\left|g\left(\eta_{2}\right)\right| d \eta_{1} d \eta_{2} \\
\leq & C\left(\int_{B} \int_{B}\left|\eta_{1}\right|^{(5-d) / 2}\left|\eta_{2}\right|^{(5-d) / 2}\left(1-\eta_{1}^{\prime} \cdot \eta_{2}^{\prime}\right)^{(3-d) / 2} d \eta_{1} d \eta_{2}\right)^{1 / 2}<\infty .
\end{aligned}
$$

When $k \geq 3$, we see that $f \in \dot{H}^{1 / 2}\left(\mathbb{R}^{d}\right)$, and by a similar argument using the CauchySchwarz inequality, we conclude that $g$ is locally integrable. 
Now, since (4.2) holds for $g$ and $G$ we have

$$
g\left(\eta_{1}\right) g\left(\eta_{2}\right)=\widetilde{G}\left(\left|\eta_{1}\right|+\left|\eta_{2}\right|, \eta_{1}+\eta_{2}\right)
$$

for almost every $\left(\eta_{1}, \eta_{2}\right) \in \mathbb{R}^{d} \times \mathbb{R}^{d}$, where $\widetilde{G}$ is equal to $G$ modulo composition with certain translations and multiplication by nonzero scalars (depending on $g$ ). The continuity of $g$ and $G$ now follows from Lemma 7.20 (which in fact holds for all $d \geq 2$ ) and Proposition 7.5 of [13].

Hence, it suffices to characterise all solutions to the functional equation

$$
g\left(\eta_{1}\right) g\left(\eta_{2}\right)=G\left(\left|\eta_{1}\right|+\left|\eta_{2}\right|, \eta_{1}+\eta_{2}\right) \quad \text { for each }\left(\eta_{1}, \eta_{2}\right) \in \mathbb{R}^{d} \times \mathbb{R}^{d},
$$

where $g$ and $G$ are continuous. In this case, we may assume $g(0) \neq 0$. Otherwise (4.4) gives

$$
G(|\eta|, \eta)=g(\eta) g(0)=0
$$

for all $\eta \in \mathbb{R}^{d}$, which, combined with (4.4) again, implies that

$$
g(\eta)^{2}=G(2|\eta|, 2 \eta)=G(|2 \eta|, 2 \eta)=0
$$

for all $\eta \in \mathbb{R}^{d}$, and this is the trivial case.

Noting that $G(0,0)=g(0)^{2} \neq 0$, we can rewrite (4.4) as

$$
H\left(\left|\eta_{1}\right|, \eta_{1}\right) H\left(\left|\eta_{2}\right|, \eta_{2}\right)=H\left(\left|\eta_{1}\right|+\left|\eta_{2}\right|, \eta_{1}+\eta_{2}\right) \quad \text { for each }\left(\eta_{1}, \eta_{2}\right) \in \mathbb{R}^{d} \times \mathbb{R}^{d},
$$

where $H(\tau, \xi)=G(0,0)^{-1} G(\tau, \xi)$. By algebraic properties of the cone (see [13, Lemma 7.18]), this implies

$$
H(X) H(Y)=H(X+Y) \quad \text { for all } X, Y \in\left\{(\tau, \xi) \in \mathbb{R}^{d+1}: \tau>|\xi|\right\} .
$$

Thus, by [13, Lemma 7.1], there exist $a \in \mathbb{C}$ and $b \in \mathbb{C}^{d}$ such that

$$
H(\tau, \xi)=\exp (a \tau+b \cdot \xi)
$$

for $(\tau, \xi)$ in the solid cone. Choosing $c \in \mathbb{C}$ such that $\exp (2 c)=G(0,0)$, we obtain $G(\tau, \xi)=\exp (a \tau+b \cdot \xi+2 c)$, so that $g(\xi)=\exp (a|\xi|+b \cdot \xi+c)$. Thus, the Fourier transforms of the maximisers $f$ take the form

$$
\widehat{f}(\xi)=|\xi|^{-1} \exp (a|\xi|+b \cdot \xi+c) .
$$

It remains to check under which conditions on $a$ and $b$ the right-hand side of (2.1), or equivalently the quantity (4.3), is finite. It is easy to see that $\operatorname{Re}(a)<0$ is necessary and $c \in \mathbb{C}^{d}$ has no effect on such considerations. So we assume $\operatorname{Re}(a)<0$ and $c=0$ from now on, and consider the cases $\alpha(k)=0$ and $\alpha(k)>0$ separately.

When $\alpha(k)=0$ the quantity (4.3) is equal to the $k$ th power of

$$
\|f\|_{\dot{H}^{1 / 2}\left(\mathbb{R}^{d}\right)}^{2}=\int_{\mathbb{R}^{d}} \exp (2 \operatorname{Re}(a)|\xi|+2 \operatorname{Re}(b) \cdot \xi) \frac{d \xi}{|\xi|} .
$$


In polar coordinates this is equal to a constant multiple of

$$
\int_{-1}^{1} \int_{0}^{\infty} \exp ((2 \operatorname{Re}(a)+2|\operatorname{Re}(b)| u) r) r^{d-2}\left(1-u^{2}\right)^{(d-3) / 2} d r d u,
$$

which is finite if and only if $|\operatorname{Re}(b)|<-\operatorname{Re}(a)$.

When $\alpha(k)>0$, the quantity (4.3) is bounded above by

$$
\|f\|_{\dot{H}^{1 / 2}\left(\mathbb{R}^{d}\right)}^{2(k-2)}\|f\|_{\dot{H}^{(\alpha(k)+1) / 2}\left(\mathbb{R}^{d}\right)}^{4}
$$

and using polar coordinates as above it is easy to check that if $|\operatorname{Re}(b)|<-\operatorname{Re}(a)$ then $\|f\|_{\dot{H}^{s}\left(\mathbb{R}^{d}\right)}<\infty$ for any $s \geq 1 / 2$. Also, we have shown above that if $|\operatorname{Re}(b)| \geq-\operatorname{Re}(a)$ then $\|f\|_{\dot{H}^{1 / 2}\left(\mathbb{R}^{d}\right)}$ is not finite, and therefore the quantity (4.3) is not finite if $k \geq 3$. Hence it remains to show that for $k=2, d \geq 4$ and $|\operatorname{Re}(b)| \geq-\operatorname{Re}(a)$ the quantity (4.3) is not finite. In this case, (4.3) is equal to

$$
\begin{aligned}
& \int_{\mathbb{R}^{d}} \int_{\mathbb{R}^{d}} \exp \left(2 \operatorname{Re}(a)\left|\eta_{1}\right|\right.\left.+2 \operatorname{Re}(b) \cdot \eta_{1}\right) \exp \left(2 \operatorname{Re}(a)\left|\eta_{2}\right|+2 \operatorname{Re}(b) \cdot \eta_{2}\right) \\
& \cdot\left|\eta_{1}\right|^{(d-5) / 2}\left|\eta_{2}\right|^{(d-5) / 2}\left(1-\eta_{1}^{\prime} \cdot \eta_{2}^{\prime}\right)^{(d-3) / 2} d \eta_{1} d \eta_{2} .
\end{aligned}
$$

Let $\Omega \subset \mathbb{S}^{d-1}$ be a closed cap centred at $\operatorname{Re}(b)^{\prime}$ so that $\operatorname{Re}(b)^{\prime} \cdot \eta_{2}^{\prime} \geq 1-\varepsilon$ for each $\eta_{2}^{\prime} \in \Omega$. Then, for each $\eta_{1}$ with $\eta_{1}^{\prime} \in \Omega^{\perp}$, we see that the $d \eta_{2}$-integral in (4.5) is bounded below by a constant multiple of

$$
\int_{\Omega} \int_{0}^{\infty} \exp \left(\left(2 \operatorname{Re}(a)+2 \operatorname{Re}(b) \cdot \eta_{2}^{\prime}\right) r\right) r^{(3 d-7) / 2} d r d \sigma\left(\eta_{2}^{\prime}\right),
$$

which is not finite when $|\operatorname{Re}(b)| \geq-\operatorname{Re}(a)$, and hence neither is (4.3). This completes the proof Theorem 2.1.

\section{The Schrödinger equation: Carneiro's inequality revisited}

The following theorem is the natural analogue of Theorem 1.1 for the Schrödinger evolution operator and is due to Carneiro [7].

Theorem 5.1. Let $d \geq 2$. Then the inequality

$$
\left\|e^{i t \Delta} f_{1} e^{i t \Delta} f_{2}\right\|_{L_{t, x}^{2}\left(\mathbb{R}^{d+1}\right)}^{2} \leq \mathrm{S}(d, 2) \int_{\mathbb{R}^{2 d}}\left|\widehat{f}_{1}\left(\xi_{1}\right)\right|^{2}\left|\widehat{f}_{2}\left(\xi_{2}\right)\right|^{2}\left|\xi_{1}-\xi_{2}\right|^{d-2} d \xi_{1} d \xi_{2}
$$

holds with sharp constant given by

$$
\mathrm{S}(d, 2)=2^{-d}(2 \pi)^{-3 d+1}\left|\mathbb{S}^{d-1}\right|
$$

which is attained if and only if

$$
\widehat{f_{j}}(\xi)=\exp \left(a|\xi|^{2}+b \cdot \xi+c_{j}\right),
$$

where $a, c_{1}, c_{2} \in \mathbb{C}, b \in \mathbb{C}^{d}$ and $\operatorname{Re}(a)<0$. 
The case $d=1$ is special because, for $f_{1}$ and $f_{2}$ with separated Fourier support, we have the identity ${ }^{3}$

$$
\left\|e^{i t \Delta} f_{1} e^{i t \Delta} f_{2}\right\|_{L_{t, x}^{2}\left(\mathbb{R}^{1+1}\right)}^{2}=\frac{1}{2(2 \pi)^{2}} \int_{\mathbb{R}^{2}}\left|\widehat{f_{1}}\left(\xi_{1}\right)\right|^{2}\left|\widehat{f}_{2}\left(\xi_{2}\right)\right|^{2} \frac{d \xi_{1} d \xi_{2}}{\left|\xi_{1}-\xi_{2}\right|},
$$

which follows easily by changes of variables and Plancherel's theorem. This is evident from the calculation in [15, Theorem 1.2] or [28, p. 412] (see also [14, Section 17] for an analogous inequality for the extension operator on $\mathbb{S}^{1}$ ). The interaction weight is too singular for the right-hand side of (5.1) to be finite for integrable $f_{1}=\lambda f_{2} \neq 0$. A manifestation of this is that $\mathrm{S}(1,2)=(2 \pi)^{-2}$ is equal to twice the constant arising in the identity (5.1).

With $d=2$, the power of the interaction weight is zero, and so the estimate reduces to the sharp version of the Strichartz estimate (1.5) due to Foschi [13].

For the case $d=4$, Carneiro deduced the following corollary from Theorem 5.1 in the same way that the inequality of Corollary 2.2 was deduced from Theorem 2.1.

Corollary 5.2. Let $d=4$. Then

$$
\left\|e^{i t \Delta} f\right\|_{L^{4}\left(\mathbb{R}^{4+1}\right)} \leq(32 \pi)^{-1 / 4}\|f\|_{L^{2}\left(\mathbb{R}^{4}\right)}^{1 / 2}\|\nabla f\|_{L^{2}\left(\mathbb{R}^{4}\right)}^{1 / 2} .
$$

The constant is sharp and is attained if and only if

$$
\widehat{f}(\xi)=\exp \left(a|\xi|^{2}+i b \cdot \xi+c\right),
$$

where $a, c \in \mathbb{C}, b \in \mathbb{R}^{d}$ and $\operatorname{Re}(a)<0$.

Note that the class of maximisers is smaller than that of Theorem 5.1 (although in [7] it was suggested otherwise). The maximisers can be obtained from $u(0, x)=\exp \left(-|x|^{2}\right)$ under the action of the group generated by:

(S1) $u(t, x) \mapsto u\left(t+t_{0}, x+x_{0}\right)$ with $t_{0} \in \mathbb{R}, x_{0} \in \mathbb{R}^{d}$,

(S2) $u(t, x) \mapsto \lambda_{1} u\left(\lambda_{2}^{2} t, \lambda_{2} x\right)$ with $\lambda_{1}, \lambda_{2}>0$.

(S3) $u(t, x) \mapsto e^{i \theta} u(t, x)$ with $\theta \in \mathbb{R}$.

Another well-known symmetry for the Schrödinger equation is the Galilean transformation:

(S4) $u(t, x) \mapsto \exp \left(-i\left(x \cdot v+|v|^{2} t\right)\right) u(t, x+2 v)$ with $v \in \mathbb{R}^{d}$.

Foschi [13] proved that the maximisers for (1.5) with $d=1,2$ are given by

$$
\widehat{f}(\xi)=\exp \left(a|\xi|^{2}+b \cdot \xi+c\right),
$$

where $a, c \in \mathbb{C}, b \in \mathbb{C}^{d}$ and $\operatorname{Re}(a)<0$, which is a larger class than that of Corollary 5.2. This is explained by the fact that

$$
\left\|e^{i t \Delta} f\right\|_{L_{t, x}^{p}\left(\mathbb{R}^{d+1}\right)}\|f\|_{L^{2}\left(\mathbb{R}^{d}\right)}^{-1}
$$

\footnotetext{
3 The authors thank Luis Vega for bringing this to their attention.
} 
is invariant under the action of (S4), whereas

$$
\left\|e^{i t \Delta} f\right\|_{L_{t, x}^{p}\left(\mathbb{R}^{d+1}\right)}\|f\|_{L^{2}\left(\mathbb{R}^{d}\right)}^{-1 / 2}\|\nabla f\|_{L^{2}\left(\mathbb{R}^{d}\right)}^{-1 / 2}
$$

is not.

To conclude we present a $k$-linear generalisation of Theorem 5.1 which is analogous to Theorem 2.1 .

Theorem 5.3. Suppose that $d, k \geq 2$ and let $\beta(k)=d(k-1) / 2-1$. Let $K:\left(\mathbb{R}^{d}\right)^{k} \rightarrow$ $[0, \infty)$ be given by

$$
K(\eta)=\left(\sum_{1 \leq i<j \leq k}\left|\eta_{i}-\eta_{j}\right|^{2}\right)^{1 / 2}
$$

for $\eta=\left(\eta_{1}, \ldots, \eta_{k}\right) \in\left(\mathbb{R}^{d}\right)^{k}$. Then the inequality

$$
\left\|\prod_{j=1}^{k} e^{i t \Delta} f_{j}\right\|_{L_{t, x}^{2}\left(\mathbb{R}^{d+1}\right)}^{2} \leq \mathrm{S}(d, k) \int_{\mathbb{R}^{k d}} \prod_{j=1}^{k}\left|\widehat{f_{j}}\left(\eta_{j}\right)\right|^{2} K(\eta)^{2 \beta(k)} d \eta
$$

holds with sharp constant given by

$$
\mathrm{S}(d, k)=\pi(2 \pi)^{-d(2 k-1)} k^{-d k / 2+1}\left|\mathbb{S}^{(k-1) d-1}\right|
$$

which is attained if and only if

$$
|\xi| \widehat{f}_{j}(\xi)=\exp \left(a|\xi|+b \cdot \xi+c_{j}\right),
$$

where $a, c_{1}, \ldots, c_{k} \in \mathbb{C}, b \in \mathbb{C}^{d}, \operatorname{Re}(a)<0$ and $|\operatorname{Re}(b)|<-\operatorname{Re}(a)$.

Theorem 5.3 was proven by Carneiro in [7] following the argument of HundertmarkZharnitsky [16]. We remark that it can also be proven by following the proof of Foschi [13].

Acknowledgments. The authors wish to thank Jon Bennett and Thomas Duyckaerts for helpful conversations. They also thank Sanghyuk Lee and Seoul National University, where part of this research was conducted, for their warm hospitality.

The first author is supported by EPSRC grant EP/J021490/1. The second author is supported by the ERC grant 277778 and the MINECO grants MTM2010-16518 and SEV-2011-0087.

\section{References}

[1] Bahouri, H., Gérard, P.: High frequency approximation of solutions to critical nonlinear wave equations. Amer. J. Math. 121, 131-175 (1999) Z Zbl 0919.35089 MR 1705001

[2] Barceló, B.: On the restriction of the Fourier transform to a conical surface. Trans. Amer. Math. Soc. 292, 321-333 (1985) Zbl 0578.42006 MR 0805965

[3] Bégout, P., Vargas, A.: Mass concentration phenomena for the $L^{2}$-critical nonlinear Schrödinger equation. Trans. Amer. Math. Soc. 359, 5257-5282 (2007) Zbl 1171.35109 MR 2327030 
[4] Bennett, J., Bez, N., Carbery, A., Hundertmark, D.: Heat-flow monotonicity of Strichartz norms. Anal. PDE 2, 147-158 (2009) Zbl 1190.35043 MR 2547132

[5] Bourgain, J.: Estimates for cone multipliers. In: Geometric Aspects of Functional Analysis, Oper. Theory Adv. Appl. 77, Birkhäuser, 41-60 (1995) Zbl 0833.43008 MR 1353448

[6] Bulut, A.: Maximizers for the Strichartz inequalities for the wave equation. Differential Integral Equations 23, 1035-1072 (2010) Zbl 1240.35314 MR 2742477

[7] Carneiro, E.: A sharp inequality for the Strichartz norm. Int. Math. Res. Notices 2009, 31273145 Zbl 1178.35090 MR 2533799

[8] Christ, M., Shao, S.: Existence of extremals for a Fourier restriction inequality. arXiv:1006.4319.

[9] Christ, M., Shao, S.: On the extremizers of an adjoint Fourier restriction inequality. Adv. Math. 230, 957-977 (2012) Zbl pre06059016 MR 2921167

[10] Carles, R., Keraani, S.: On the role of quadratic oscillations in nonlinear Schrödinger equations. II. The $L^{2}$-critical case. Trans. Amer. Math. Soc. 359, 33-62 (2007) Zbl 1115.35119 MR 2247881

[11] Duyckaerts, T., Merle, F., Roudenko, S.: Maximizers for the Strichartz norm for small solutions of mass-critical NLS. Ann. Scuola Norm. Sup. Pisa 10, 427-476 (2011) Zbl 1247.35142 MR 2856155

[12] Fanelli, L., Vega, L., Visciglia, N.: On the existence of maximizers for a family of restriction theorems. Bull. London Math. Soc. 43, 811-817 (2011) Zbl 1225.42012 MR 2820166

[13] Foschi, D.: Maximizers for the Strichartz inequality. J. Eur. Math. Soc. 9, 739-774 (2007) Zbl 1231.35028 MR 2341830

[14] Foschi, D., Klainerman, S.: Bilinear space-time estimates for homogeneous wave equations. Ann. Sci. École Norm. Sup. (4) 33, 211-274 (2000) Zbl 0959.35107 MR 1755116

[15] Hörmander, L.: Oscillatory integrals and multipliers on FLp. Ark. Mat. 11, 1-11 (1973) Zbl 0254.42010 MR 0340924

[16] Hundertmark, D., Zharnitsky, V.: On sharp Strichartz inequalities in low dimensions. Int. Math. Res. Notices 2006, art. ID 34080, 18 pp. Zbl 1131.35308 MR 2219206

[17] Kenig, C. E., Merle, F.: Global well-posedness, scattering and blow-up for the energy critical focusing non-linear wave equation. Acta Math. 201, 147-212 (2008) Zbl 1183.35202 MR 2461508

[18] Klainerman, S., Machedon, M.: Space-time estimates for null forms and the local existence theorem. Comm. Pure Appl. Math. 46, 1221-1268 (1993) Zbl 0803.35095 MR 1231427

[19] Klainerman, S., Machedon, M.: Remark on Strichartz-type inequalities. With appendices by Jean Bourgain and Daniel Tataru. Int. Math. Res. Notices 1996, 201-220 Zbl 0853.35062 MR 1383755

[20] Klainerman, S., Machedon, M.: On the regularity properties of a model problem related to wave maps. Duke Math. J. 87, 553-589 (1997) Zbl 0878.35075 MR 1446618

[21] Kunze, M.: On the existence of a maximizer for the Strichartz inequality. Comm. Math. Phys. 243, 137-162 (2003) Zbl 1060.35133 MR 2020224

[22] Lee, S., Rogers, K. M., Vargas, A.: Sharp null form estimates for the wave equation in $\mathbb{R}^{3+1}$. Int. Math. Res. Notices 2008, art. ID rnn 096, 18 pp. Zbl 1160.35040 MR 2439536

[23] Lee, S., Vargas, A.: Sharp null form estimates for the wave equation. Amer. J. Math. 130, 1279-1326 (2008) Zbl 1158.35112 MR 2450209

[24] Merle, F., Vega, L.: Compactness at blow-up time for $L^{2}$ solutions of the critical nonlinear Schrödinger equation in 2D. Int. Math. Res. Notices 1998, 399-425 Zbl 0913.35126 MR 1628235 
[25] Ramos, J. M.: A refinement of the Strichartz inequality for the wave equation with applications. Adv. Math. 230, 649-698 (2012) Zbl pre06040344 MR 2914962

[26] Shao, S.: Maximizers for the Strichartz and the Sobolev-Strichartz inequalities for the Schrödinger equation. Electron. J. Differential Equations 2009, no. 3, 13 pp. Z Zbl 1173.35692 MR 2471112

[27] Stein, E. M.: Singular Integrals and Differentiability Properties of Functions. Princeton Univ. Press (1970) Zbl 0207.13501 MR 0290095

[28] Stein, E. M.: Harmonic Analysis. Princeton Univ. Press (1993) Zbl 0821.42001 MR 1232192

[29] Strichartz, R. S.: Restrictions of Fourier transforms to quadratic surfaces and decay of solutions of wave equations. Duke Math. J. 44, 705-714 (1977) Zbl 0372.35001 MR 0512086

[30] Tao, T.: Endpoint bilinear restriction theorems for the cone, and some sharp null form estimates. Math. Z. 238, 215-268 (2001) Zbl 0992.42004 MR 1865417

[31] Wolff, T.: A sharp bilinear cone restriction estimate. Ann. of Math. (2) 153, 661-698 (2001) Zbl 1125.42302 MR 1836285 